\title{
Desgarro completo traumático del músculo pectoral mayor. M. Pectoralis major, T. A.
}

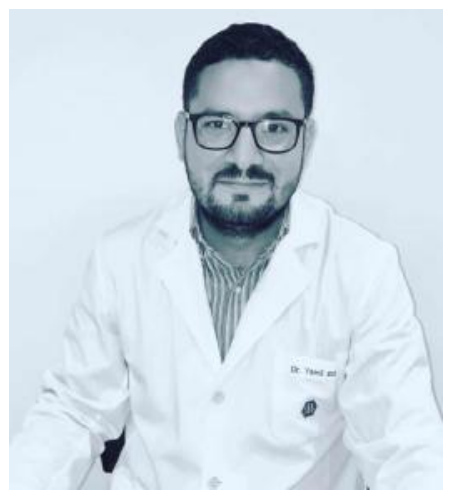

Dr. Eid Caballero Juan Yamil

Aspirante a la SBCPER

Clínica Montalvo, Servicio de Cirugía Plástica y Reconstructiva.

Santa Cruz de la Sierra, Bolivia.

yami_eid@hotmail.com

+591-70812312

\section{"Complete traumatic tear of the pectoralis major muscle"}

Palabra Claver: Rotura de pectoral mayor - toxina botulínica - malla de reemplazo. Key Words: Rupture of the pectoralis major - Botulinum toxin - mesh replacement

\section{RESUMEN}

Se presenta un caso de desgarro completo del músculo pectoral mayor (m. pectoralis major, T.A.) en un paciente fisicoculturista durante su ejercicio en banca fija. Se procedió a reconstruir en 2 etapas. Primera etapa: se colocó toxina botulínica (200 U.I.). Segunda etapa: a los 21 días se procedió a la reparación quirúrgica de la zona con la colocación de malla de doble capa (polipropileno y politetrafluoroetileno). Se obtuvo excelente recuperación funcional y estética.

\section{INTRODUCCIÓN}

La avulsión del pectoral mayor es una lesión muy poco frecuente. El primer caso fue publicado en 1822 por Patissier (1) y desde entonces no más de 130 casos fueron reportados en la literatura médica.

Se produce mayormente en deportistas de alto rendimiento o en fisicoculturistas, por un movimiento brusco de contracción excéntrica con el hombro en abducción y rotación externa (2).

El objetivo del presente trabajo es presentar un caso con rotura completa del pectoral mayor (m. pectoralis major, T.A.) en su

\section{ABSTRACT}

A case of complete rupture of the pectoralis major muscle (m. pectoralis major, TA) in a bodybuilder patient during the exercise is presented. We carry out the reconstruction in 2 steps. First step: botulinum toxin (200 U.I.) in the muscle was injected. Second step: 21 days post-inyections we done a surgical repair with placing a double layer mesh (polypropylene and polytetrafluoroethylene mesh). Excellent functional and aesthetic recovery was obtained.

cuerpo muscular, su tratamiento y la resolución del problema con restitución ad-integrum tanto funcional como estética.

\section{PRESENTACIÓN DEL CASO}

En el año 2013 se presentó a la consulta un paciente de 30 años de edad sexo masculino derivado del Servicio de Cirugía Toráxica con diagnóstico de desgarro completo del músculo pectoral mayor izquierdo ( $m$. pectoralis major, T.A.).

Refiere el paciente que durante su actividad deportiva en el 
gimnasio hace 6 meses atrás presentó un desplazamiento de las pesas mientras realizaba ejercicios en banca plana. Esto provocó la rotación externa del brazo y hombro izquierdo con inmediato dolor y formación de hematoma en la zona del tercio externo con los dos tercios internos del pectoral mayorizquierdo. El paciente fue evaluado por el Servicio de traumatología donde se le indicó hielo local, así como analgésicos-antiinflamatorios. A los 2 meses del trauma, se le realizaron estudios ecográficos
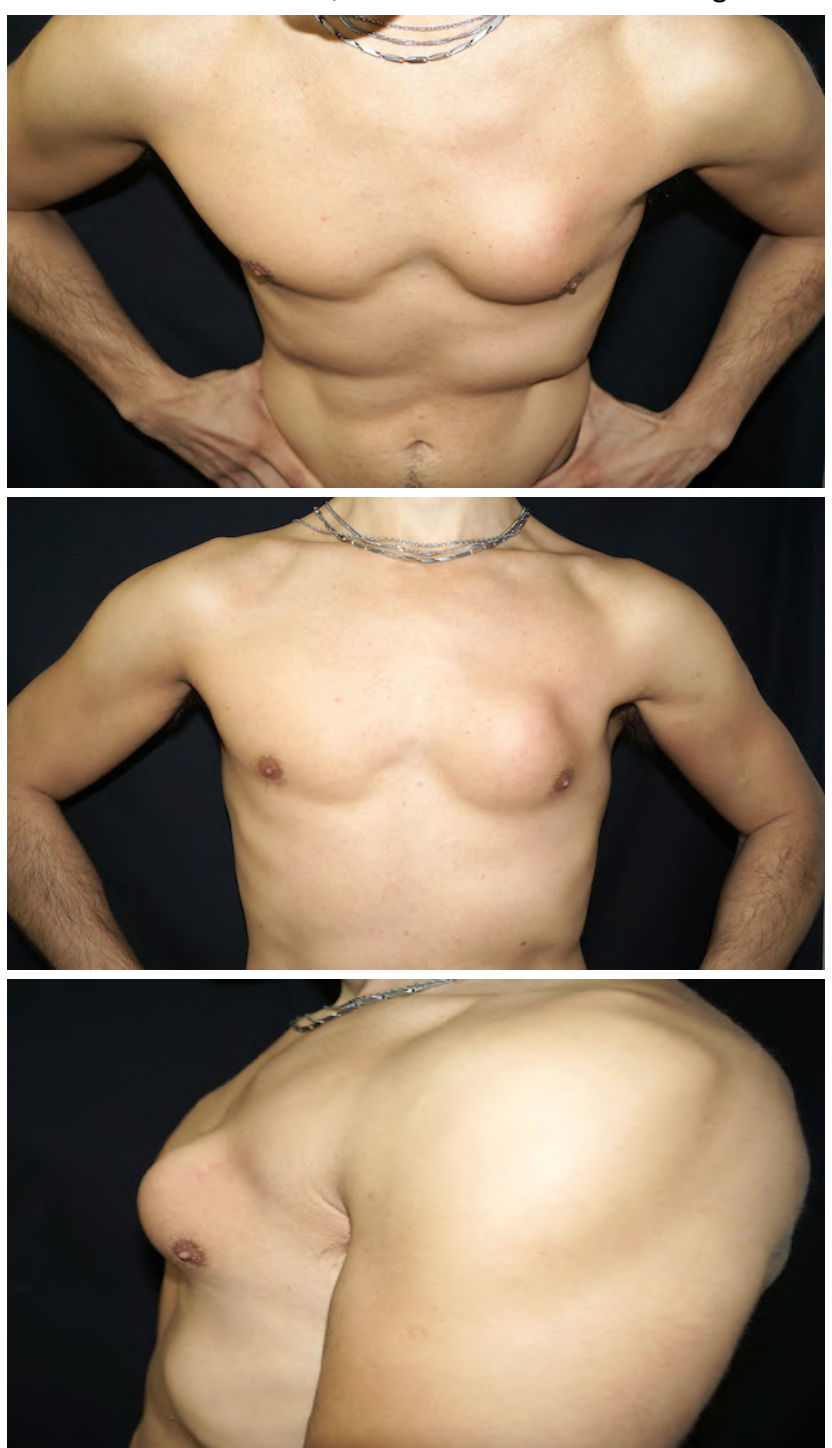

FOTOS 1-3: Imágenes preoperatorias (distorsión de los haces del pectoral mayor durante la contracción)

donde se demostró la sección del pectoral mayor izquierdo. Durante los siguientes 4 meses el paciente fue controlado por traumatología y cirugía de tórax, y ante la deformación estética debida a la contracción del músculo, fue derivado a nuestro Servicio para eventual resolución quirúrgica.

En la decisión quirúrgica se tomó en cuenta la edad, la actividad física del paciente (deportista de alto rendimiento - fisicoculturismo) y el deseo por regresar a su aspecto físico previo al trauma. El plan de tratamiento fue dividido en 2 etapas: primera etapa se planificó la relajación del músculo pectoral y en la segunda etapa se ideó la reparación de los cabos seccionados del pectoral con el soporte de una malla de refuerzo interna.

\section{TÉCNICA QUIRÚRGICA}

Primera etapa: Se colocó toxina botulínica (marca Botox) 200 U.I. sobre los haces musculares próximos al esternón y cartílagos costales izquierdos. La distribución de la toxina fue profunda, seleccionando los puntos a $2 \mathrm{~cm}$ cada uno entre sí y en la zona de máxima contracción (FOTO 4).

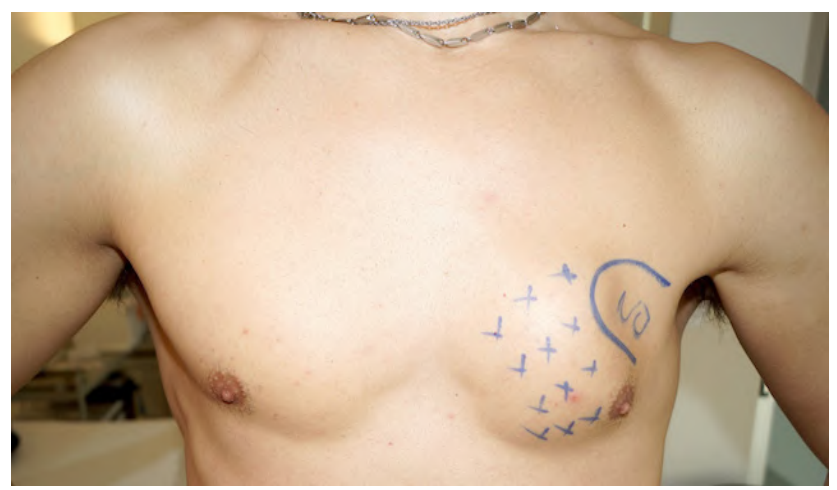

FOTO 4: Colocación de 200 UI de toxina botulínica en los haces del pectoral mayor para relajar musculatura en forma química pre quirúrgica.

Segunda etapa: Se programó a los 21 días post colocación de la toxina botulínica la resolución quirúrgica con el objetivo de unir los cabos seccionados musculares y reforzarlos con
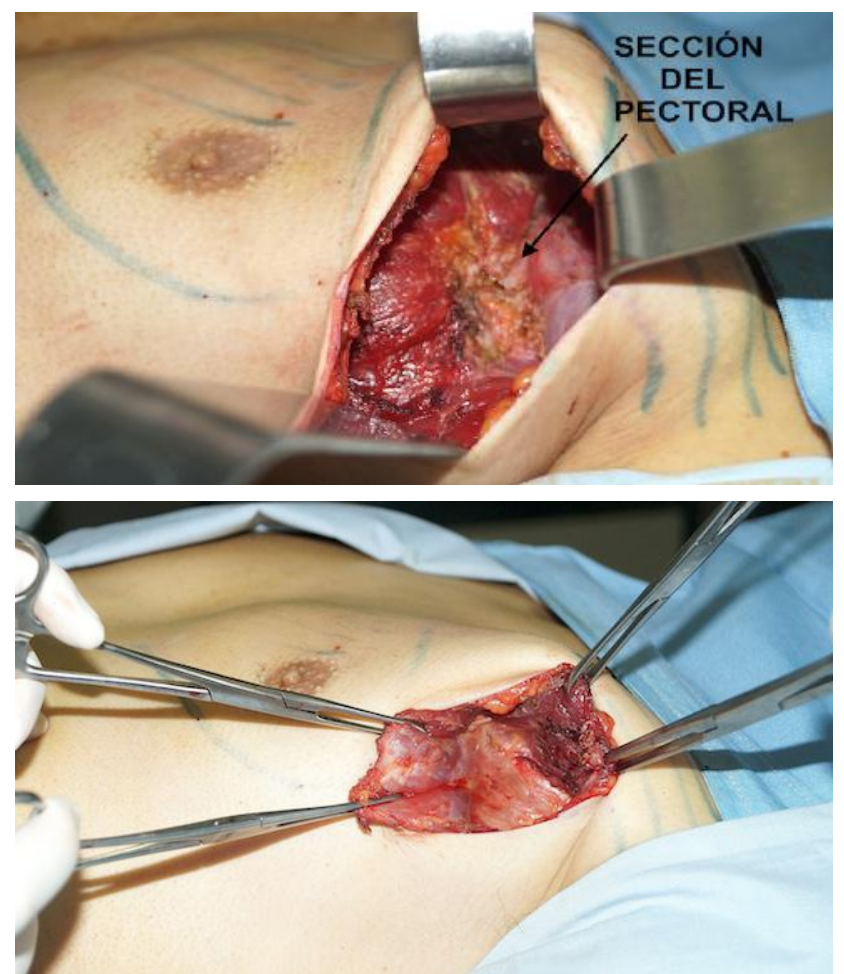

FOTO 5 Y 6: Imágenes intraoperatorias donde se observa la sección completa del músculo pectoral mayor 
una malla en forma posterior. El acto quirúrgico se realizó bajo anestesia general. Se infiltró la zona con una solución anestésica de $20 \mathrm{ml}$ de lidocaína al $2 \%$ con epinefrina, diluida en $250 \mathrm{ml}$ de solución fisiológica al 0,9\% y $1 \mathrm{ml}$ de adrenalina a una concentración de 1:1000. Se realizó el abordaje lateral de la zona sobre el borde del pectoral mayor izquierdo en busca de la solución de continuidad del músculo. Se identificó la zona seccionada donde se constató presencia de tejido cicatrizal y hundimiento de la misma.
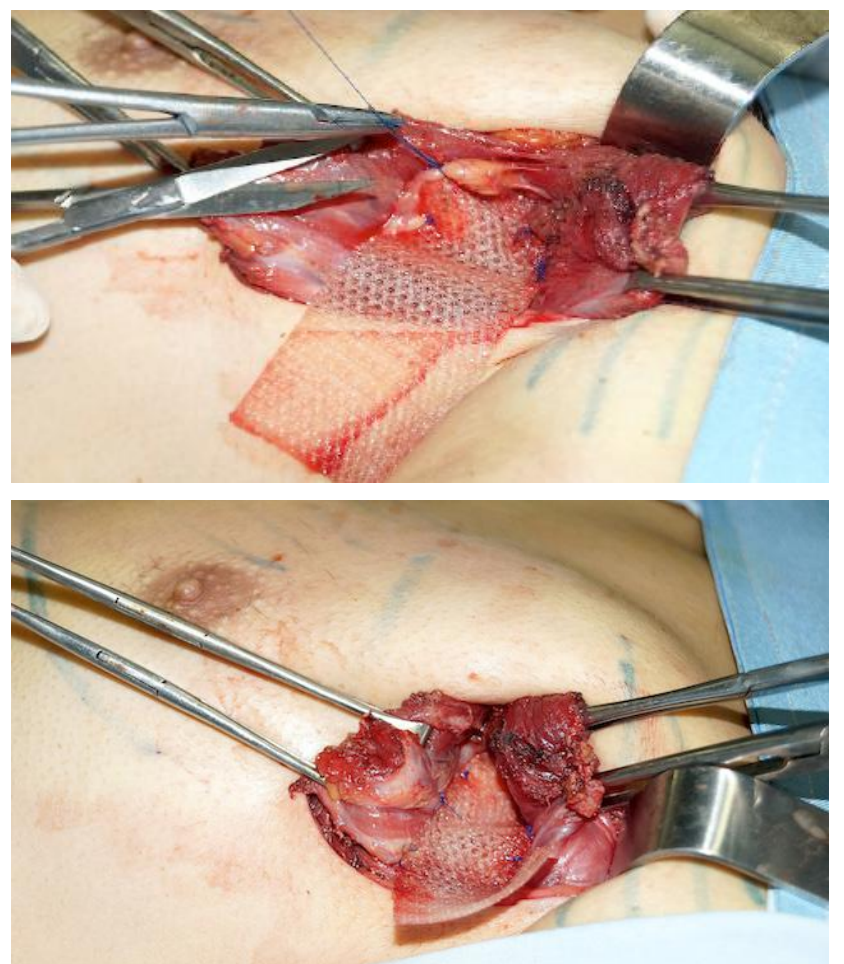

FOTOS 7 Y 8: Imágenes intraoperatorias donde se observa la colocación de malla de doble capa en la cara posterior del m. pectoral mayor.

A continuación, se colocó en el plano posterior intermuscular (entre elm. pectoral mayory pectoral menor) (m. pectoralis major y m. pectoralis minor, T.A.) una malla de doble capa, marca Bard Composix constituída por una lámina de PTFE expandido y otra de polipropileno. Esta última capa fue colocada en dirección a la cara profunda del pectoral mayor uniendo los bordes seccionados. Dicha malla fue fijada en posición con material irreabsorbible (polipropileno 1.0).

Se realizó control de hemostasia, cierre por plano de forma directa con aproximación de los cabos de sección del pectoral mayor con polipropileno 1.0, curación plana y colocación de goma espuma en la zona para hacer compresión suave, evitando los hematomas. Se inmovilizó el miembro de la región afectada mediante un cabestrillo tipo Vietnam durante 30 días. Primera curación a las 48 hrs y retiro de puntos a los 20 días de la cirugía.

\section{RESULTADOS}

Durante la evolución postoperatoria el paciente no presentó complicaciones tales como hematoma, infección, así como
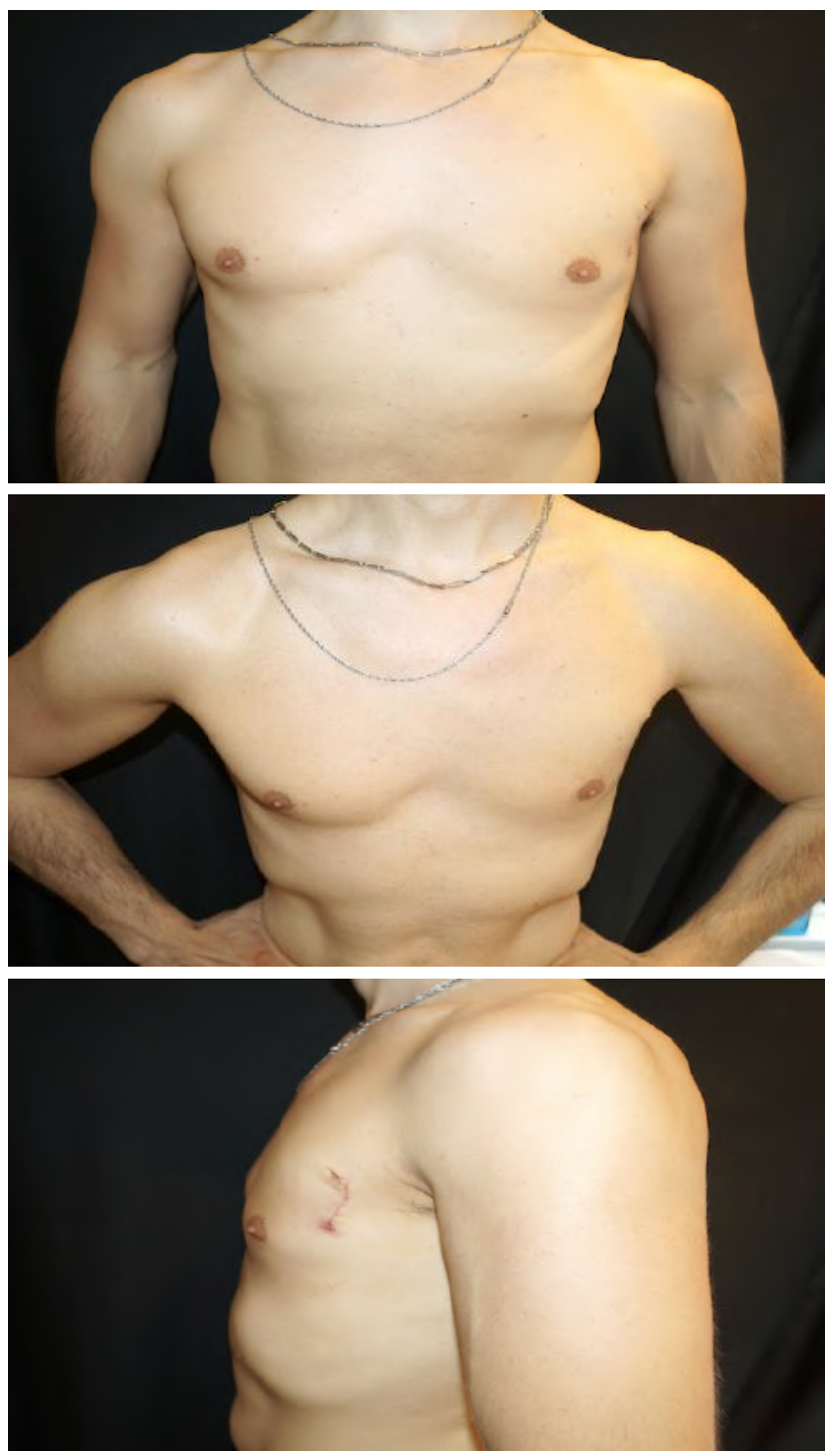

FOTOS. 9-10 Y 11: Imágenes postoperatorias en reposo y contracción a los 60 días con reconstitución de wlas fibras seccionadas

tampoco mostró alteraciones de la cicatriz. El paciente fue controlado a las 48 hrs y al día $5,15,20,30$,60 con evolución favorable y recuperación total.

A los 30 días fue derivado al servicio de fisiokinesioterapia para la rehabilitación y fortalecimiento muscular progresivo. No ha presentado recidiva de la lesión, comenzando su actividad deportiva a los 90 días. La restitución funcional fue completa con gran satisfacción por parte del paciente.

\section{DISCUSIÓN}

La ruptura del pectoral mayor es una lesión que se ha incrementado en los últimos tiempos debido al aumento de las actividades en gimnasio, sumado al aumento de la sobrecarga 


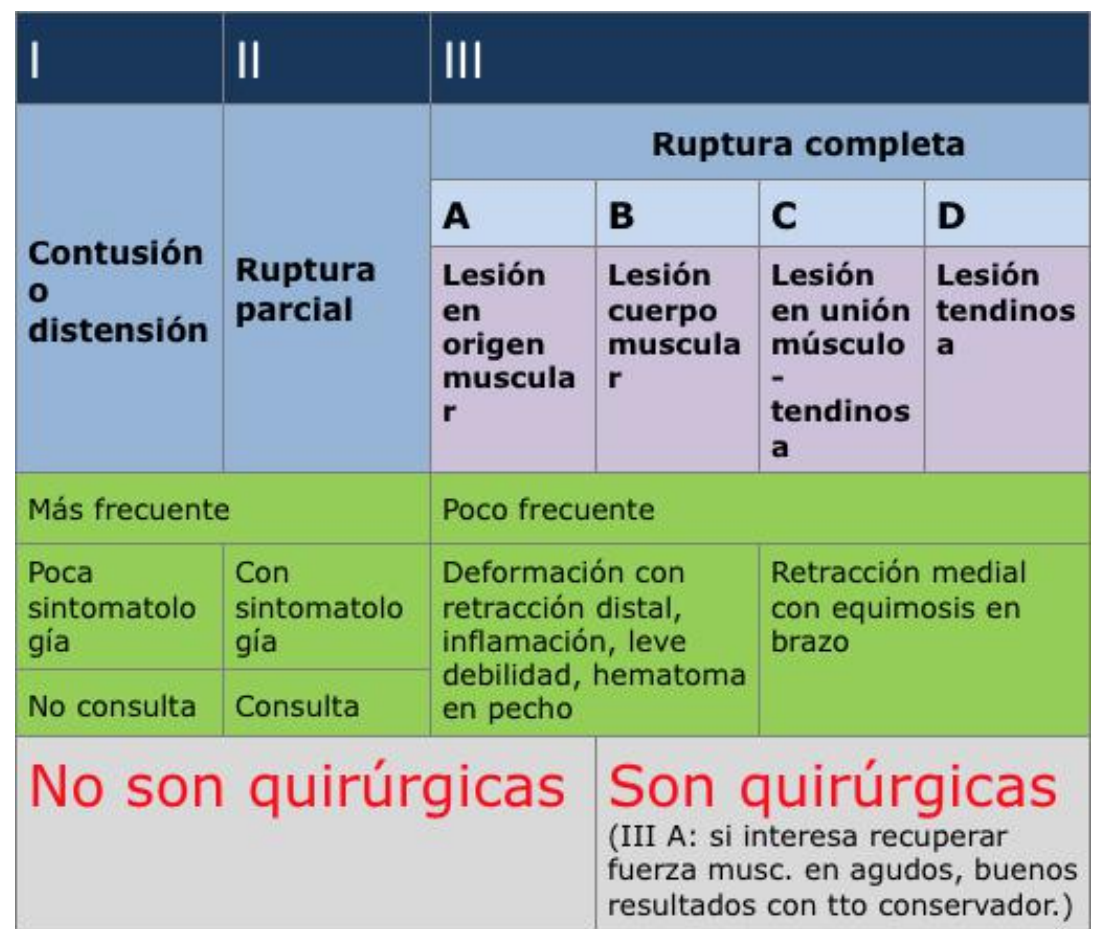

Tabla.01-Clasificación de Tietjen (Tietjen, Ronald A. Close Injuries of the Pectoralis Mayor Muscle. The Joumal of Trauma, 20:262-264, 1980)

del trabajo debido al uso de anabólicos-esteroides. Estos últimos provocan una hipertrofia muscular con debilitamiento de las fibras siendo más propensos a este tipo de lesiones. El mecanismo de sección es por sobre esfuerzo en máxima contracción excéntrica del miembro superior con el brazo en abducción o extensión intentando aducción.

Tietjen (3) clasificó en 1980 a las lesiones cerradas del pectoral mayor en 3 grupos (Ver. Tabla 01)

\section{Grupo I: Contusión o distensión}

Grupo Il: Rotura parcial

Grupo III: Rotura completa, las cuales son de resolución quirúrgica.

El diagnóstico primario es clínico confirmado por estudios de imágenes principalmente por ecografía y/o resonancia magnética nuclear que nos permite clasificar la lesión.

El tratamiento depende de la edad del paciente, del tipo de actividad, si es aguda o crónica, la ubicación y tipo de lesión: parcial o completa. El tratamiento conservador es válido, aunque los reportes son pocos, su principal indicación son niños, ancianos, pacientes sedentarios y lesiones parciales. Para los demás pacientes con rupturas completas la indicación del tratamiento es quirúrgica por debilidad muscular, dolor crónico y demanda física estética (4-9).

En nuestro planteamiento del paso se decidió colocar toxina botulínica con el objeto de relajar los haces musculares del pectoral y evitar la retracción del mismo permitiendo un cierre sin tensión. Por otra parte, se evaluó la colocación de una malla para refuerzo de la sutura de la zona desgarrada. Dentro de las diversas mallas, creemos aconsejable el empleo de las de doble capa permitiendo la capa polipropileno una adherencia firma y bien integrada a la región posterior muscular (biointegración) y una capa no adherente conformada por el politetrafluoroetileno con el objeto de no adherirse a la superficie del pectoral menor y permitir un deslizamiento sin adherencias.

\section{CONCLUSIÓN}

La sección de las fibras del pectoral mayor está descripta con cierta frecuencia en pacientes fisicoculturistas o pacientes con gran actividad deportiva. La sección completa requiere resolución quirúrgica para restituir la funcionalidad y la estética a la zona del tórax. En nuestro caso, presentamos un paciente en el cual se relajó previo a la cirugía los haces musculares con toxina botulínica y posteriormente se procedió a la colocación de una malla de doble capa con el objeto de reforzar la sutura muscular.

En nuestra pesquisa bibliográfica no hemos encontrado descrita la técnica de colocación de malla ni la de colocación de toxina botulínica para reconstrucción del pectoral mayor.

\section{BIBLIOGRAFÍA}

1. Patissier $P$ (1882) Traite des maladies des artisans. Paris 162-164. 
2. Dr. Stumbo Daniel, Dr. Alberto Arnoldo, Dr. Acuña Aníbal, Dr. Gotter Guillermo, Dr. Salome Héctor. Ruptura del pectoral mayor. ¿Cuándo opero? Revista de la Asociación Argentina de Traumatología del Deporte, 1(6): 36-41,2013

3. Tietjen, Ronald A. Close Injuries of the Pectoralis Major Muscle. The Journal of Trauma, 20:262-264, 1980.

4. Antii, Alho. Ruptured Pectoralis Major Muscle. Acta Orthop Scand. 65 (6):652-653, 1994.)

5. Lindenbaun, Barry. Delayed Repair of a Ruptured Pectoralis Major Muscle. Clinical Orthop. 109:120-121, 1975.

6. Wolfe, Scott; Wickiewicz, Thomas; Cavanaugh, John. Ruptures of the Pectoralis Major Muscle. The American Journal of Sports Medicine. No. 5, Vol. 20: 587-593, 1992.)

7. Liu Jain; Jiunn-Jer Wu; Cheng-Yen Chang; Yi-Hong Chou; Wai-Hee Lo. Avulsion of the Pectoralis Major Tendon. The American Journal of Sports Medicine. No. 3, Vol 20: 366368, 1992.)

8. Brunelli, M.; Gilí, Thomas; Fracturas y Lesiones Tendinosas del Hombro del Deportista. The Orthop Clin of North America. No. 3:527-528, 2002

9. Anbari, Ammar; Kelly, John and Mover, Ray. Delayed Repair of a Ruptured Pectoralis Major Muscle. The American Journal of Sports Medicine. No.2, Vol.28: 254-256, 2000 